\title{
SUPERFLUIDITY IN THE STOCHASTIC LIMIT
}

\author{
L. ACCARDI AND S. V. KOZYREV
}

Received 5 January 2004

We discuss the stochastic limit approach to superfluidity. Starting from the usual Hamiltonian describing the interaction between the Bose condensate and the normal phase, we prove the existence of superfluidity in the stochastic limit and deduce a nonlinear (quadratic) kinetic equation describing the evolution of the density of superfluid liquid.

\section{Introduction}

The theory of superfluidity was developed by Landau and Bogolyubov (cf. $[10,11,14]$ ), for an introduction see [12]. An analogue of the approach of [10,11] was applied to superconductivity theory (cf. [13]).

The essence of the superfluidity phenomenon is that the Bose condensate becomes superfluid and friction between condensate and normal phase disappears. Bogolyubov in $[10,11]$ found that this can be explained as an effect of stabilization of the condensate by interaction between particles.

Since the main point of superfluidity is the absence of quantum dissipation, it is natural to expect that the stochastic limit of quantum theory, which is the mathematical theory of quantum dissipation and transport (cf. [8]), should play a role in the description of this phenomenon.

In the present paper, using as a starting point the approach of $[10,11]$, we introduce a Hamiltonian that describes the interaction between the Bose condensate and the normal phase and investigate this Hamiltonian using the stochastic limit approach.

The name stochastic limit is due to the property that in this limit, the quantum fields become quantum white noises and the Schrödinger equation becomes a white noise Hamiltonian equation. The main advantage of this new technique is that this limit preserves all the relevant physical information in the scale of interest and at the same time essentially simplifies the mathematical description so to allow to extract this information and put it to use. 


\section{Superfluidity in the stochastic limit}

The starting point of the weak coupling version of the stochastic limit is a Hamiltonian of the form

$$
H=H_{0}+\lambda H_{I}
$$

where $\lambda$ is a coupling constant. One then makes the time rescaling $t \rightarrow t / \lambda^{2}$ in the solution $U_{t}^{(\lambda)}=e^{i t H_{0}} e^{-i t H}$ of the Schrödinger equation in interaction picture associated to the Hamiltonian $H$, that is,

$$
\frac{\partial}{\partial t} U_{t}^{(\lambda)}=-i \lambda H_{I}(t) U_{t}^{(\lambda)}, \quad U_{0}^{(\lambda)}=1
$$

with $H_{I}(t)=e^{i t H_{0}} H_{I} e^{-i t H_{0}}$ (the evolved interaction Hamiltonian). This leads to the rescaled equation

$$
\frac{\partial}{\partial t} U_{t / \lambda^{2}}^{(\lambda)}=-\frac{i}{\lambda} H_{I}\left(\frac{t}{\lambda^{2}}\right) U_{t / \lambda^{2}}^{(\lambda)}
$$

Then one proves the existence of the limits (in a topology specified in [8])

$$
\lim _{\lambda \rightarrow 0} H_{I}\left(\frac{t}{\lambda^{2}}\right)=H_{t}, \quad \lim _{\lambda \rightarrow 0} U_{t / \lambda^{2}}^{(\lambda)}=U_{t}
$$

Moreover, one proves that $U_{t}$ is the solution of the white noise Hamiltonian equation

$$
\partial_{t} U_{t}=-i H_{t} U_{t}, \quad U_{0}=1
$$

and that this equation is equivalent to a quantum stochastic differential equation. We refer to [8] for further discussion of the stochastic limit approach.

The structure of the present paper is as follows. In Section 2, we discuss the Bogolyubov-Landau condition of superfluidity. In Sections 3 and 4, we review some basic facts on Bogolyubov's approach to Bose condensation in ideal and nonideal Bose gases. In Section 5, we introduce the Hamiltonian of condensate-normal phase interaction and investigate this model using the stochastic limit. In Section 6, we construct by the stochastic golden rule the master equation for the condensate-normal phase interaction and prove the existence of superfluidity in this system. We prove that the standard BogolyubovLandau condition is not sufficient to guarantee the superfluidity in the stochastic limit and introduce a procedure to overcome this problem. In Section 7, we sum up the main conclusion that can be drawn from our results.

\section{The Landau and Bogolyubov ideas on superfluidity theory}

Tissa and London conjectured that the existence of the Bose condensate can explain superfluidity in the sense that the condensate corresponds to the superfluid component and the temperature phase corresponds to normal component.

The following heuristic argument, due to Landau [14], suggests that there should be no superfluidity in ideal Bose gas. 
Suppose that the condensate is moving with velocity $u$ and the normal state is static. Suppose that the friction of the condensate with the normal phase stimulates the transition of a particle in the condensate from the state with velocity $u$ to a state with velocity $u-p / m$. Then this particle will have energy $(\mu-p)^{2} / 2 m$. Before the transition, the particle had energy $\mu^{2} / 2$. Since the transition is due to friction with normal state, it is accompanied by the excitation of a particle of the normal phase with energy $p^{2} / 2 m$. We get the energy difference

energy after transition + energy of excitation - energy before transition

$$
=\frac{(\mu-p)^{2}}{2 m}+\frac{p^{2}}{2 m}-\frac{\mu^{2}}{2}=\frac{p^{2}}{m}-p u<0 \quad \text { for small } p \text {. }
$$

This means that such transitions are energetically possible and that the condensate is unstable.

To overcome this difficulty, Bogolyubov introduced interaction into this picture and found $[10,11]$ that the interaction between the particles in the condensate may stabilize the condensate by modifying the energy difference in such a way that transitions to the slower motion, due to friction, will proceed with positive energy difference and therefore will be energetically forbidden.

Bogolyubov found that interaction makes the state of normal phase instable.

To stabilize the condensate, one makes a Bogolyubov transformation which changes the dispersion $E(k)$ of excitations of normal phase (cf. [10, 11, 12, 13]). More precisely, after the canonical transformation, the energy difference for transition $p \mapsto p-k$ is given by

$$
E(k)+\varepsilon(p-k)-\varepsilon(p)
$$

where $\varepsilon(p)=p^{2} / 2 m$ and $E(p)$ has different dependence on $p$ (cf. (4.20)). In this case, one can overcome the Landau objection. Consider the following examples.

Example 2.1. The radiative dispersion in the nonrelativistic approximation is $E(k)=c|k|$. In this case, (2.2) takes the form

$$
c|k|-p k+\frac{1}{2} k^{2}>0, \quad|p|<c
$$

which is always satisfied if $|p|<c$. Notice that, for the validity of the above argument, the specific form of the radiative dispersion is not essential: it is only needed that $E(k)>|k|$.

Example 2.2. The dispersion in the polaron model is $E(k)=\omega$. In this case, (2.2) becomes

$$
\omega-k p+\frac{1}{2} k^{2}>0, \quad|p|<\sqrt{2 \omega}
$$

We show that the results of Landau and Bogolyubov discussed above are related with the stochastic limit approach. One of the important results of the stochastic limit is that the main properties of the dynamics are controlled by $\delta$-functions of energy differences 
6 Superfluidity in the stochastic limit

of the form

$$
\delta(E(k)+\varepsilon(p-k)-\varepsilon(p))
$$

(which is precisely the $\delta$-function applied to the expression (2.2)). The possibility to suppress dissipation by exploiting such $\delta$-functions was called in [4] the Cheshire cat effect. We will prove that the Bogolyubov-Landau condition of superfluidity is in fact strictly related to the Cheshire cat effect developed in the stochastic limit approach.

\section{Condensation of an ideal Bose gas}

In the present section, we discuss standard material on Bose condensation of an ideal Bose gas (cf. [12]). The Hamiltonian of an ideal Bose gas in second quantization is given by

$$
H=\int \omega(p) b^{*}(p) b(p) d p
$$

where $b^{*}(p), b(p)$ are boson operators: $\left[b(p), b^{*}\left(p^{\prime}\right)\right]=\delta\left(p-p^{\prime}\right)$. The grand canonical ensemble Hamiltonian is

$$
\Gamma=H-\mu N, \quad N=\int b^{*}(p) b(p) d p .
$$

The equilibrium state of the gas is the unique mean zero gauge invariant state with covariance

$$
\left\langle b^{*}(p) b(p)\right\rangle=\langle n(p)\rangle=\frac{1}{e^{\beta(\omega(p)-\mu)}-1}
$$

giving the density of particles with momentum $p$ and where $\beta=1 / \theta$ is the inverse temperature. We take $\mu \leq 0$ since $\langle n(p)\rangle \geq 0$ for all $p$. The integral, over all momenta, gives the global density of the gas:

$$
\int\langle n(p)\rangle d p=\rho
$$

To describe Bose condensation, Bogolyubov considers the zero mode of the field $b_{0}$ as singled out from the other modes and satisfying

$$
\left[b_{0}, b_{0}^{*}\right]=1 \text {. }
$$

This is equivalent to taking, as the reference state of the field, not the equilibrium state but a mean zero Gaussian state with variance

$$
\left\langle b^{*}(p) b\left(p^{\prime}\right)\right\rangle=\langle n(p)\rangle \delta\left(p-p^{\prime}\right)=c \delta(p)+\frac{1}{e^{\beta \omega(p)}-1} \delta\left(p-p^{\prime}\right) .
$$

Notice that the chemical potential in this formula is taken equal to zero. 
We choose the constant $c=c(\theta)$ depending on the temperature so that

$$
\langle n(p)\rangle=\rho\left(1-\left(\frac{\theta}{\theta_{c}}\right)^{3 / 2}\right) \delta(p)+\frac{1}{e^{\beta \omega(p)}-1} .
$$

To determine the constant $\theta_{c}$, we write the total density of the gas as the sum of the density of the condensate plus the density of the normal phase:

$$
\rho=c+\int \frac{1}{e^{\beta \omega(p)}-1} d p
$$

Therefore, by the conservation of number of particles,

$$
c=\rho\left(1-\left(\frac{\theta}{\theta_{c}}\right)^{3 / 2}\right)
$$

where $d=3$ and $\omega(p)=p^{2} / 2 m, \theta \leq \theta_{c}$. The critical temperature $\theta_{c}$ is the temperature when the integral in (3.8) becomes equal to the total density of particles $\rho$ and condensate disappears.

Remark 3.1. The above discussion indicates that any mean zero gauge invariant state with covariance (3.6) incorporates a phase transition at the kinematical level, that is, independently of the dynamics. One fixes the density $\rho$ and defines $\theta_{c}$ by the condition $c\left(\theta_{c}\right)-0$. All this is however at a merely kinematical level: the dispersion of the Hamiltonian is still as in (3.1) and therefore, Landau's instability objection still applies. In the following section, we outline Bogolyubov's argument to overcome the difficulty by exploiting the role of a nontrivial interaction.

\section{Condensation of a nonideal Bose gas}

In the present section, we review material from [12] which discusses the Bose condensation of a nonideal Bose gas and proves the applicability of the Bogolyubov-Landau condition of stability of the condensate.

The second quantized Hamiltonian of a nonideal Bose gas is a modification of the Hamiltonian (3.1) with the introduction of a chemical potential $\mu$ and a quartic term:

$$
\begin{aligned}
\Gamma= & \sum_{p}(\omega(p)-\mu) b^{*}(p) b(p) \\
& +\frac{\lambda}{2 V} \sum_{p_{1}+p_{2}=p_{1}^{\prime}+p_{2}^{\prime}} g\left(p_{1}-p_{1}^{\prime}\right) b^{*}\left(p_{1}\right) b^{*}\left(p_{2}\right) b\left(p_{2}^{\prime}\right) b\left(p_{1}^{\prime}\right) .
\end{aligned}
$$

We consider a state such that almost all particles with $p=0$ are in the condensate. This allows to simplify the Hamiltonian $\Gamma$.

Since $b(p)$ with $p \neq 0$ are not in the condensate, the amplitudes of $b(p)$ with $p \neq 0$ are small with respect to $b_{0}$. Therefore, we can keep in the Hamiltonian $\Gamma$ only the terms of second order in $b(p)$ with $p \neq 0$ and neglect the higher- (i.e., 3rd- and 4th-) order terms. 
This approximation will be valid only when almost all particles are in the condensate, that is, for a temperature $\theta$ close to zero.

In this approximation, the fourth-order term in $\Gamma$ takes the form

$$
\begin{aligned}
& \frac{\lambda}{2 V}\left(g(0) N_{0}^{2}+b_{0}^{* 2} \sum_{p} g(p) b(p) b(-p)+b_{0}^{*} b_{0} \sum_{p}(g(p)+g(-p)) b(p) b(p)\right. \\
& \left.\quad+2 b_{0}^{*} b_{0} \sum_{p} g(0) b(p) b(p)+b_{0}^{2} \sum_{p} g(p) b^{*}(p) b^{*}(-p)\right)
\end{aligned}
$$

Introducing the additional condition $g(p)=g(-p)$ and using $\omega(0)=0$, we get for $\Gamma$,

$$
\begin{aligned}
\Gamma= & \frac{\lambda N_{0}^{2}}{2 V} g(0)-\mu N_{0}+\sum_{p \neq 0}\left(\omega(p)-\mu+\frac{\lambda N_{0}}{V} g(0)+\frac{\lambda N_{0}}{V} g(p)\right) b^{*}(p) b(p) \\
& +\frac{\lambda}{2 V} b_{0}^{* 2} \sum_{p} g(p) b(p) b(-p)+\frac{\lambda}{2 V} b_{0}^{2} \sum_{p} g(p) b^{*}(p) b^{*}(-p) .
\end{aligned}
$$

This implies that the ground state of $\Gamma$ has energy

$$
E_{1}=\frac{\lambda N_{0}^{2}}{2 V} g(0)-\mu N_{0}
$$

The chemical potential is defined by the equation

$$
\frac{\partial E_{1}}{\partial N_{0}}=0
$$

hence in our case, it is equal to

$$
\mu=\frac{\lambda N_{0}}{V} g(0)
$$

The assumption that almost all particles with $p=0$ are in the condensate implies that

$$
\left\langle b_{0}^{*} b_{0}\right\rangle=N_{0}, \quad N_{0} \gg 1,
$$

where we denote by $b_{0}^{*}$ the creation of the particle with $p=0$. Since

$$
\left\langle b_{0} b_{0}^{*}\right\rangle=N_{0}+1
$$

and $N_{0}+1$ is almost equal to $N_{0}$, we can consider $b_{0}$ as a classical (commuting) variable with

$$
\left|b_{0}\right|^{2}=b_{0}^{*} b_{0}=b_{0}^{*} b_{0}=N_{0}, \quad b_{0}=\sqrt{N_{0}} e^{i \phi} .
$$


With this chemical potential and making the canonical transformation $b(p) \mapsto b(p) e^{i \phi}$, we cancel the phase of the complex number $b_{0}=\sqrt{N_{0}} e^{i \phi}$ so that the Hamiltonian $\Gamma$ becomes

$$
\begin{aligned}
\Gamma= & -\frac{\lambda N_{0}^{2}}{2 V} g(0)+\sum_{p \neq 0}\left(\omega(p)+\frac{\lambda N_{0}}{V} g(p)\right) b^{*}(p) b(p) \\
& +\frac{\lambda N_{0}}{2 V} \sum_{p \neq 0} g(p)\left(b^{*}(p) b^{*}(-p)+b(-p) b(p)\right) .
\end{aligned}
$$

The quadratic Hamiltonian (4.10) is diagonalized by the following canonical transformation:

$$
\begin{gathered}
a(p)=u_{p} b(p)+v_{p} b^{*}(-p), \\
a^{*}(p)=u_{p} b^{*}(p)+v_{p} b(-p), \\
u_{p}^{2}-v_{p}^{2}=1, \quad u_{p}=u_{-p}, v_{p}=v_{-p}, u_{p}, v_{p} \in \mathbb{R} .
\end{gathered}
$$

The inverse transformation of this is given by

$$
\begin{gathered}
b(p)=u_{p} a(p)-v_{p} a^{*}(-p), \\
b^{*}(p)=u_{p} a^{*}(p)-v_{p} a(-p) .
\end{gathered}
$$

After this canonical transformation, the off-diagonal terms in the Hamiltonian (i.e., the coefficients of $a^{*}(p) a^{*}(-p)$ and $\left.a(-p) a(p)\right)$ become equal to

$$
-u_{p} v_{p}\left(\omega(p)+\frac{\lambda N_{0}}{V} g(p)\right)+\left(u_{p}^{2}+v_{p}^{2}\right) \frac{\lambda N_{0}}{2 V} g(p)
$$

and the diagonal terms (coefficients of $\left.a^{*}(p) a(p)\right)$ are equal to

$$
\left(u_{p}^{2}+v_{p}^{2}\right)\left(\omega(p)+\frac{\lambda N_{0}}{V} g(p)\right)-4 u_{p} v_{p} \frac{\lambda N_{0}}{2 V} g(p) .
$$

Since $u_{p}^{2}-v_{p}^{2}=1$, we can use the hyperbolic parametrization

$$
u_{p}=\operatorname{ch} x, \quad v_{p}=\operatorname{sh} x
$$

We impose the vanishing of the off-diagonal terms (4.13) through the so-called "compensation equation" which, in the above parametrization, takes the form

$$
\text { th } 2 x=\frac{\left(\lambda N_{0} / V\right) g(p)}{\omega(p)+\lambda\left(N_{0} / V\right) g(p)} \text {. }
$$


This implies

$$
\begin{gathered}
u_{p}^{2}+v_{p}^{2}=\operatorname{ch} 2 x=\frac{1}{\sqrt{1-\left(\left(\left(\lambda N_{0} / V\right) g(p)\right) /\left(\omega(p)+\left(\lambda N_{0} / V\right) g(p)\right)\right)^{2}}}, \\
2 u_{p} v_{p}=\operatorname{sh} 2 x=\frac{1}{\sqrt{1-\left(\left(\left(\lambda N_{0} / V\right) g(p)\right) /\left(\omega(p)+\left(\lambda N_{0} / V\right) g(p)\right)\right)^{2}}} \frac{\left(\lambda N_{0} / V\right) g(p)}{\omega(p)+\left(\lambda N_{0} / V\right) g(p)} .
\end{gathered}
$$

Under the above conditions, using (4.14), we see that the Hamiltonian takes the form

$$
\Gamma=-\frac{\lambda N_{0}^{2}}{2 V} g(0)+\sum_{p \neq 0} E(p) a^{*}(p) a(p)
$$

which is of the same form as that of the ideal Bose gas, but with a new dispersion $E(p)$, due to interaction and given by

$$
E(p)=\sqrt{\omega^{2}(p)+\frac{2 \lambda N_{0}}{V} \omega(p) g(p)} .
$$

The condition $g(0)>0$, that provides positivity of the value under the square root, corresponds to the domination of repulsion (cf. [13]).

The state for the new Hamiltonian (4.18) is obtained from (3.6) simply by replacing the $a$-field for the $b$-field.

The Bogolyubov-Landau energy balance condition (2.2) for the Hamiltonian (4.18) becomes

$$
E(p)+\frac{m(u-p / m)^{2}}{2}-\frac{\mu^{2}}{2}=E(p)-p u+\frac{p^{2}}{2 m}
$$

The Bogolyubov-Landau argument used for Example 2.1 is now applicable to (4.20) since $E(p)>c|p|$ and, for small $p$ and for $u<c$, we can get a situation when the energy of excitation is positive and transitions from condensate to normal state proceed with consumption of energy so that the condensate is stable.

\section{Condensate-normal phase interaction in the stochastic limit}

In the present section, we will discuss how to describe the Bogolyubov-Landau condition by a stochastic limit approach which improves the Bogolyubov discussion by considering the $p=0$ modes, not as a scalar degree of freedom, but as a (slow) Boson quantum field.

An intermediate approximation, in which the terms $b_{0}^{* 2}, b_{0}^{2}, N_{0}:=b_{0}^{*} b_{0}$ in (4.3) come from a 1-mode quantum field, will be discussed elsewhere. 
We will consider the stochastic limit of a system with initial state given by (3.6). We will see that the associated phase transition leads to the emergence of nonlinear kinetic equations. This shows that phase transition and the stochastic limit procedure do not commute: to investigate a system with phase transition by the stochastic limit, we should describe first the phase transition.

The present approach is close to the discussion in Abrikosov-Gorkov-Dzialoshinskii book [1]. "Consider the Bose liquid with zero temperature that flows with velocity $\mathbf{v}$ In the presence of friction in the liquid, there will be elementary excitations with different velocities." An analysis of these elementary excitations gives the Bogolyubov-Landau condition (cf. $[1,12,14])$.

We introduce the Hamiltonian for condensate-normal state interaction and investigate it in the stochastic limit.

Consider a system with two Bose fields (phases)

$$
\left[c(p), c^{+}\left(p^{\prime}\right)\right]=\delta\left(p-p^{\prime}\right), \quad\left[a(k), a^{+}\left(k^{\prime}\right)\right]=\delta\left(k-k^{\prime}\right) .
$$

We describe the condensate (which, in the stochastic limit terminology, plays the role of "system" or "slow degrees of freedom") by the Bose field $c(p)$, labeled by a velocity index $p$, and with free Hamiltonian

$$
H_{c}=\int \varepsilon(p) c^{*}(p) c(p) d^{3} p
$$

with dispersion

$$
\varepsilon(p)=\frac{m p^{2}}{2}
$$

and state

$$
\left\langle c^{*}(p) c\left(p^{\prime}\right)\right\rangle=N(p) \delta\left(p-p^{\prime}\right)
$$

which describes the distribution of the condensate density over velocities.

The second Bose field $a(p)$ ("reservoir" or "fast" degrees of freedom) describes the excitations of the nonideal Bose gas (normal phase) which diagonalize the interaction considered in Section 4. These excitations correspond to pairs of particles with opposite momenta and have free Hamiltonian

$$
H_{n s}=\int E(p) a^{*}(p) a(p) d^{3} p
$$

The state of $a(p)$ is an equilibrium state

$$
\left\langle a^{*}(k) a\left(k^{\prime}\right)\right\rangle=\delta\left(k-k^{\prime}\right) \frac{1}{e^{\beta \omega(k)}-1} .
$$

The total Hamiltonian will be

$$
H=H_{c}+H_{n s}+\lambda H_{I}
$$




\section{Superfluidity in the stochastic limit}

with the interaction

$$
H_{I}=\iint \overline{f(k, p)} c^{*}(p) c(p-k) a(k) d^{3} p d^{3} k+\text { h.c. },
$$

where $f(u, p)$ is the form-factor (complex-valued test function).

After this, we apply to the Hamiltonian (5.7) the stochastic limit approach: by the stochastic golden rule, we construct the master equation for the density of particles in the condensate $c^{*}(p) c(p)$ and prove that the stochastic golden rule gives the BogolyubovLandau condition for this system.

The friction is due to the interaction term (5.8). The rescaled free evolution of the interaction (5.8) (of the collective field) is equal to

$$
\begin{aligned}
\mathscr{A}_{\lambda}(p, k, t) & =\frac{1}{\lambda} e^{i t H_{0} / \lambda^{2}} c^{+}(p) a(k) c(p-k) e^{-i t H_{0} / \lambda^{2}} \\
& =\frac{1}{\lambda} c^{+}(p) a(k) c(p-k) e^{-i t E(p, k) / \lambda^{2}}, \\
\mathscr{A}_{\lambda}^{+}(p, k, t) & =\frac{1}{\lambda} e^{i t H_{0} / \lambda^{2}} c^{+}(p-k) a^{+}(k) c(p) e^{-i t H_{0} / \lambda^{2}} \\
& =\frac{1}{\lambda} c^{+}(p-k) a^{+}(k) c(p) e^{i t E(p, k) / \lambda^{2}},
\end{aligned}
$$

where

$$
E(p, k)=E(k)+\varepsilon(p-k)-\varepsilon(p)
$$

is the corresponding energy difference.

The difference of energies (5.11) coincides with the difference of energies in the Bogolyubov-Landau condition (4.20) that justify the fact that Hamiltonian (5.7) describe the nonideal Bose gas considered above.

The stochastic limit of the rescaled evolution of the interaction (5.9) gives rise to a quantum white noise (master field):

$$
\lim _{\lambda \rightarrow 0} \mathscr{A}_{\lambda}(p, k, t)=B(p, k, t)
$$

After the stochastic limit, we cannot separate in the master field the degrees of freedom of reservoir and normal state. This means that the degrees of freedom of the condensate and of the normal phase become entangled even at kinematical level (cf. [2]).

The master field $B(p, k, t)$ will satisfy the module variant of the quantum Boltzmann statistics given by the following theorem.

Theorem 5.1. The entangled noise algebra is generated by $B_{1}(p, k, t), B_{2}(p, k, t)$, and $n(p)$, where

$$
B(p, k, t)=B_{1}(p, k, t)+B_{2}^{*}(p, k, t)
$$


with relations

$$
\begin{gathered}
B_{1} B_{2}^{*}=B_{2} B_{1}^{*}=0, \\
B_{1}(p, k, t) B_{1}^{*}\left(p^{\prime}, k^{\prime}, t^{\prime}\right)=2 \pi \delta\left(t-t^{\prime}\right) \delta(E(p, k)) n(p)(N(p-k)+1) \delta\left(p-p^{\prime}\right) \frac{\delta\left(k-k^{\prime}\right)}{1-e^{-\beta \omega(k)}}, \\
B_{2}(p, k, t) B_{2}^{*}\left(p^{\prime}, k^{\prime}, t^{\prime}\right)=2 \pi \delta\left(t-t^{\prime}\right) \delta(E(p, k)) n(p-k)(N(p)+1) \delta\left(p-p^{\prime}\right) \frac{\delta\left(k-k^{\prime}\right)}{e^{\beta \omega(k)}-1}, \\
{\left[n\left(p^{\prime}\right), B_{1}^{\mp}(p, k, t)\right]=( \pm)\left(\delta\left(p^{\prime}-p\right)-\delta\left(p^{\prime}-p+k\right)\right) B_{1}^{\mp}(p, k, t),} \\
{\left[n\left(p^{\prime}\right), B_{2}^{\mp}(p, k, t)\right]=(\mp)\left(\delta\left(p^{\prime}-p\right)-\delta\left(p^{\prime}-p+k\right)\right) B_{2}^{\mp}(p, k, t),} \\
{\left[n(p), n\left(p^{\prime}\right)\right]=0 .}
\end{gathered}
$$

Here $B_{i}^{-}=B_{i}, B_{i}^{+}=B_{i}^{*}$.

The zero-temperature version of this theorem was obtained in [2]. The one-particle sector relations $(n(p)=1)$ were investigated in $[5,7,9]$.

Consider now the evolution in the stochastic limit for the considered Hamiltonian. By the stochastic golden rule (cf. [8]), we get the following theorem.

THEOREM 5.2. The stochastic differential equation for $U_{t}$ has the form

$$
\begin{aligned}
d U_{t}=\left(-i \int d k d p\right. & (\bar{f}(k, p) d B(p, k, t) \\
& \left.\left.+d B^{\dagger}(p, k, t) f(k, p)\right)-d t(f \mid f)_{-}-d t \overline{(f \mid f)_{+}}\right) U_{t}
\end{aligned}
$$

where the stochastic differentials satisfy the Ito table

$$
\begin{aligned}
& d B(p, k, t) d B^{\dagger}\left(p^{\prime}, k^{\prime}, t\right)= 2 \pi n(p) \delta(\omega(k)+\varepsilon(p-k)-\varepsilon(p)) \\
& \times(N(p-k)+1) \delta\left(p-p^{\prime}\right) \frac{\delta\left(k-k^{\prime}\right)}{1-e^{-\beta \omega(k)}} d t, \\
& d B^{\dagger}(p, k, t) d B\left(p^{\prime}, k^{\prime}, t\right)= 2 \pi n(p-k) \delta(\omega(k)+\varepsilon(p-k)-\varepsilon(p)) \\
& \times(N(p)+1) \delta\left(p-p^{\prime}\right) \frac{\delta\left(k-k^{\prime}\right)}{e^{\beta \omega(k)}-1} d t, \\
&(f \mid f)_{-}=\int d k d p|f(k, p)|^{2} \frac{-i n(p)(N(p-k)+1)}{\omega(k)+\varepsilon(p-k)-\varepsilon(p)-i 0} \frac{1}{1-e^{-\beta \omega(k)}}, \\
&(f \mid f)_{+}=\int d k d p|f(k, p)|^{2} \frac{-i n(p-k)(N(p)+1)}{\omega(k)+\varepsilon(p-k)-\varepsilon(p)-i 0} \frac{1}{e^{\beta \omega(k)}-1} .
\end{aligned}
$$

The one particle version of this quantum stochastic differential equation was obtained and investigated in [6]. 


\section{Stability conditions for the condensate}

In the present section, we consider the master equation for the condensate density. The master equation is the equation for the expectation over the degrees of freedom of the reservoir (normal state in the considered case) of the Heisenberg dynamics for some observable $X$ :

$$
\frac{d}{d t} X_{t}=\frac{d\left\langle U_{t}^{*} X U_{t}\right\rangle}{d t}=\frac{d}{d t}\left\langle j_{t}(X)\right\rangle=\left\langle j_{t}\left(\theta_{0}(X)\right)\right\rangle
$$

with $U_{t}$ defined by Theorem 5.2.

If we choose $X$ to be the density $n(p)=c^{*}(p) c(p)$, the stochastic golden rule, combined with Theorems 5.2 and 5.1, gives

$$
\begin{aligned}
\frac{d}{d t} n_{t}(q)= & \int d k d p|f(k, p)|^{2}(\delta(q-p)-\delta(q-p+k)) 2 \pi \delta(E(k)+\varepsilon(p-k)-\varepsilon(p)) \\
& \times\left(n_{t}(p-k)(N(p)+1) \frac{1}{e^{\beta E(k)}-1}-n_{t}(p)(N(p-k)+1) \frac{1}{1-e^{-\beta E(k)}}\right) .
\end{aligned}
$$

Taking the integral over $p$, one gets

$$
\begin{aligned}
\frac{d}{d t} n_{t}(q)=2 \pi \int d k( & |f(k, q)|^{2} \delta(E(k)+\varepsilon(q-k)-\varepsilon(q)) \\
& \times\left(n_{t}(q-k)(N(q)+1) \frac{1}{e^{\beta E(k)}-1}-n_{t}(q)(N(q-k)+1) \frac{1}{1-e^{-\beta E(k)}}\right) \\
& -|f(k, q+k)|^{2} \delta(E(k)+\varepsilon(q)-\varepsilon(q+k)) \\
& \left.\times\left(n_{t}(q)(N(q+k)+1) \frac{1}{e^{\beta E(k)}-1}-n_{t}(q+k)(N(q)+1) \frac{1}{1-e^{-\beta E(k)}}\right)\right) .
\end{aligned}
$$

We discuss the connection of the Bogolyubov-Landau condition with (6.3). The stability of the superfluid phase corresponds to a constant density in all momenta. A sufficient condition for this is the vanishing of the two $\delta$-functions in (6.2).

Since the dispersion (4.19) for small $|k|$ is radiative $E(k)=c|k|$ and $\varepsilon(p)=p^{2} / 2 m$, we get

$$
\begin{gathered}
E(k)+\varepsilon(q-k)-\varepsilon(q)=c|k|-\frac{1}{m} q k+\frac{k^{2}}{2 m}>0, \quad \frac{1}{m}|q|<c, \\
E(k)+\varepsilon(q)-\varepsilon(q+k)=c|k|-\frac{1}{m} q k-\frac{k^{2}}{2 m}>0, \quad \frac{1}{m}|q|<c \text { and small }|k|,
\end{gathered}
$$

which shows that the stability conditions for the condensate are satisfied for $|q|<\mathrm{cm}$ and small $|k|$. More precisely, the initial densities $n_{0}(q)=n(q)$ and $N(q)$ and the form factor $f(k, q)$ can be chosen so that the right-hand side of $(6.3)$ is identically zero. We conjecture that the same result can be obtained independently of the form factor $f(k, q)$, that is, of the geometry of the problem, but a proof of this statement depends on a more detailed analysis of the "dissipation surfaces" (6.4). 


\section{A quadratic kinetic equation for the condensate}

In this section, we give a heuristic deduction of a nonlinear (quadratic) kinetic equation describing the evolution of the density of superfluid liquid. This is related to a quantum extension of the Uehling-Uhlenbeck equation and a rigorous derivation, in a more general context, is discussed in [3].

Since $N(p) \gg 1$, we can identify $N$ and $N+1$. Next we identify $n_{t}(p)$ with its expectation $\langle n(p)\rangle=N(p)$ in (6.3) (we substitute $N(p)$ by $n_{t}(p)$ in this formula). The identification

$$
\langle n(p)\rangle=N(p):=n_{t}(p)
$$

means that we consider the stochastic limit with the state depending on time in the slow time scale of the stochastic limit. This modifies the stochastic limit procedure in order to take into account the effects of phase transition.

This condition may be justified by the following argument. Equation (6.1) for $X=$ $n(q)$ is

$$
\frac{d}{d t}\left\langle U_{t}^{*} n(q) U_{t}\right\rangle=\left\langle j_{t}\left(\theta_{0}(n(q))\right)\right\rangle
$$

where $\theta_{0}(n(q))$ is equal to the right-hand side of (6.3). Then we apply the identification (7.1) for $t=0$ to (7.2). We get, in the integral on right-hand side of (7.2), combinations of terms of the following form:

$$
j_{t}(n(q) n(q-k))=j_{t}(n(q)) j_{t}(n(q-k))
$$

since $j_{t}$ is a homomorphism. After this, we apply the semiclassical approximation

$$
\left\langle j_{t}(n(q)) j_{t}(n(q-k))\right\rangle=\left\langle j_{t}(n(q))\right\rangle\left\langle j_{t}(n(q-k))\right\rangle=n_{t}(q) n_{t}(q-k)
$$

that justifies (7.1) for any $t$.

Condition (7.1) introduces the self-interaction into the considered model and makes (6.3) nonlinear. Condition (7.1) implies for (6.3) the following:

$$
\begin{aligned}
\frac{d}{d t} n_{t}(q)=-2 \pi \int d k( & |f(k, q)|^{2} \delta(E(k)+\varepsilon(q-k)-\varepsilon(q)) n_{t}(q) n_{t}(q-k) \\
& \left.-|f(k, q+k)|^{2} \delta(E(k)+\varepsilon(q)-\varepsilon(q+k)) n_{t}(q) n_{t}(q+k)\right) .
\end{aligned}
$$

We note that this equation is nonlinear (quadratic).

For (7.5), the condition of superfluidity is reduced to (6.4). This implies that (7.5) describes a superfluid flow. We get that in the stochastic limit, the condition

$$
\operatorname{Supp} n(q) \subset\{q:|q| \leq m c\}
$$

is sufficient for superfluidity in the sense that, under this condition, the right-hand side of (7.5) is zero and the density $n_{t}(q)$, of the condensate, is constant. 
We call (7.5) the equation of motion of superfluid liquid.

The nonlinear master equation (7.5) is an example of a general phenomenon. Application of the stochastic limit to systems with phase transitions will generally create nonlinear master and kinetic equations. Nonlinearity will enter through the self-interaction given by an analogue of (7.1) for a certain phase (an analogue of the condensate considered in the present paper).

\section{Conclusions}

We summarize our main results as follows:

(1) the Bogolyubov-Landau condition of superfluidity can be deduced from the stochastic limit approach;

(2) the stochastic limit approach gives not only a condition of superfluidity, but also a natural candidate for the equation of motion of the superfluid liquid (7.5);

(3) this equation is nonlinear (quadratic);

(4) nonlinear master equations should be a general feature of the stochastic limit of self-interacting systems.

\section{Acknowledgments}

The authors are grateful to I. V. Volovich and H.-D. Doebner for discussions. Sergei Kozyrev is grateful to Centro Vito Volterra and Luigi Accardi for kind hospitality. This work was partially supported by INTAS 9900545 Grant. Sergei Kozyrev was partially supported by RFFI 990100866 Grant. The work was done within the research activities of the European Union Research Training Network: "Quantum Probability with Applications to Physics, Information Theory, and Biology.”

\section{References}

[1] A. A. Abrikosov, L. P. Gorkov, and I. E. Dzialoshinskii, Methods of Quantum Field Theory in Statistical Physics, Nauka, Moscow, 1962.

[2] L. Accardi, I. Ya. Aref'eva, and I. V. Volovich, Nonequilibrium quantum field theory and entangled commutation relations, Proc. Steklov Inst. Math. 228 (2000), 106-125, dedicated to the 90th birth day of N. N. Bogolyubov.

[3] L. Accardi, S. V. Kozyrev, and A. N. Pechen, The stochastic limit of the non ideal Bose gas and the Uehling-Uhlenbeck equation, preprint, 2003.

[4] L. Accardi, S. V. Kozyrev, and I. V. Volovich, Dynamics of dissipative two-level systems in the stochastic approximation, Phys. Rev. A 56 (1997), no. 4, 2557-2562.

[5] Dynamical q-deformation in quantum theory and the stochastic limit, J. Phys. A 32 (1999), no. 19, 3485-3495.

[6] Non-exponential decay for polaron model, Phys. Lett. A 260 (1999), no. 1-2, 31-38.

[7] L. Accardi, Y. G. Lu, and I. V. Volovich, Interacting Fock Spaces and Hilbert Module Extensions of the Heisenberg Commutation Relations, Publications of IIAS, Kyoto, 1997.

[8]_, Quantum Theory and Its Stochastic Limit, Springer-Verlag, Berlin, 2002.

[9] L. Accardi, I. V. Volovich, and S. V. Kozyrev, Stochastic approximation for a model of a particle interacting with a quantum field, and a new algebra, Teoret. Mat. Fiz. 116 (1998), no. 3, 401-416 (Russian).

[10] N. N. Bogolyubov, A contribution to the theory of super-fluidity, Bull. Acad. Sci. URSS. Sér. Phys. [Izvestia Akad. Nauk SSSR] 11 (1947), 77-90 (Russian). 
[11]_, Lectures on Quantum Statistics, Radianska Shkola, Kiev, 1949.

[12] N. N. Bogolyubov and N. N. Bogolyubov Jr., Introduction to Quantum Statistical Mechanics, Nauka, Moscow, 1984 (Russian).

[13] N. N. Bogolyubov, V. V. Tolmachev, and D. V. Shirkov, A new method in the theory of superconductivity, Fortschr. Phys. 6 (1958), 605-682.

[14] L. D. Landau, On the theory of supefluidity of Helium II, J. Phys. (USSR) 11 (1947), 91.

L. Accardi: Centro Vito Volterra, Università di Roma “Tor Vergata," 00133 Roma, Italy

E-mail address: accardi@volterra.mat.uniroma2.it

S. V. Kozyrev: N. N. Semenov Institute of Chemical Physics, Russian Academy of Science, Kosygina Street, 117334 Moscow, Russia

E-mail address: kozirev@mi.ras.ru 


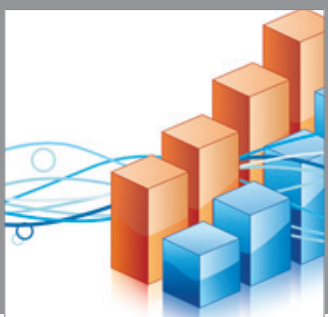

Advances in

Operations Research

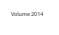

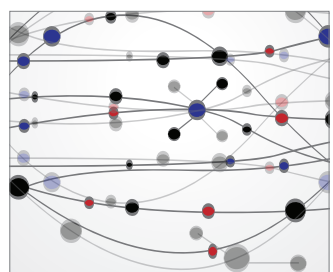

\section{The Scientific} World Journal
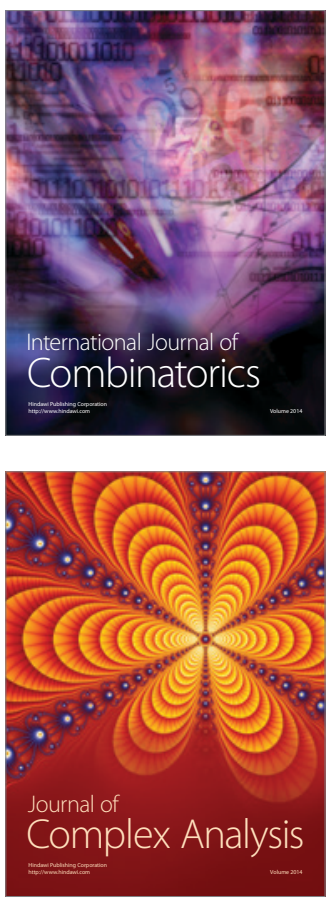

International Journal of

Mathematics and

Mathematical

Sciences
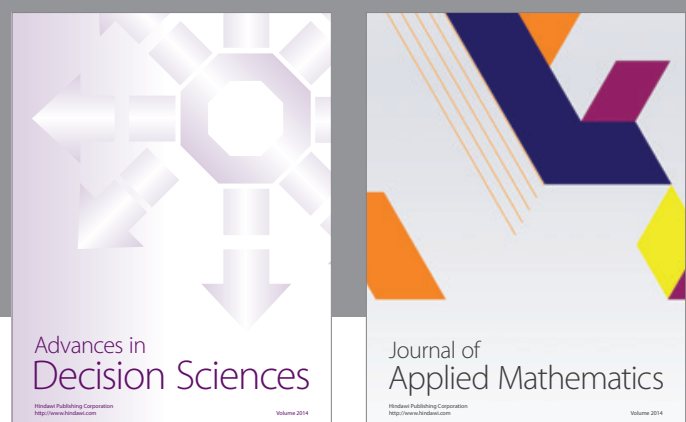

Journal of

Applied Mathematics
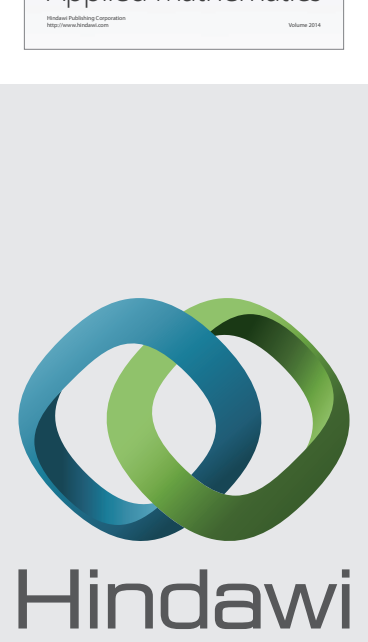

Submit your manuscripts at http://www.hindawi.com
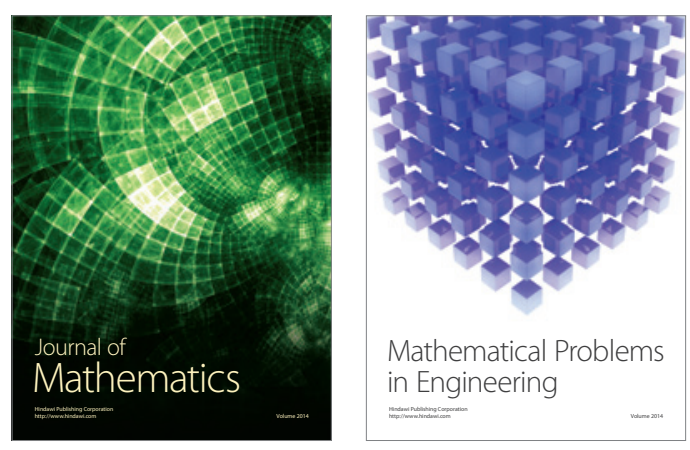

Mathematical Problems in Engineering
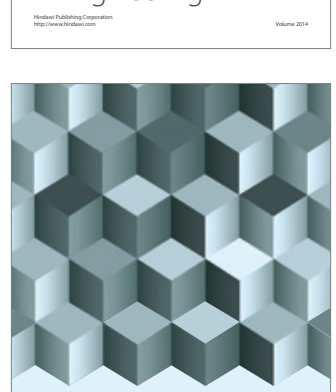

Journal of

Function Spaces
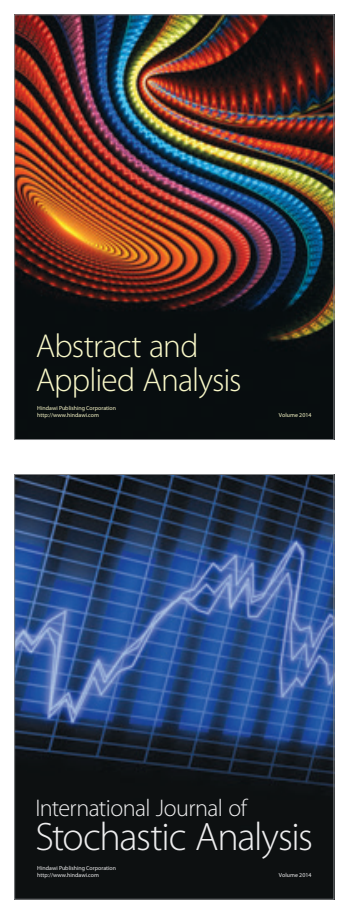

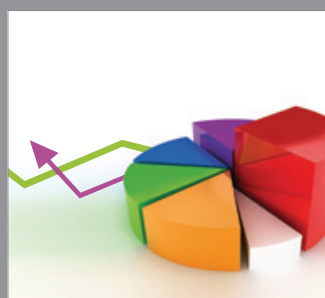

ournal of

Probability and Statistics

Promensencen
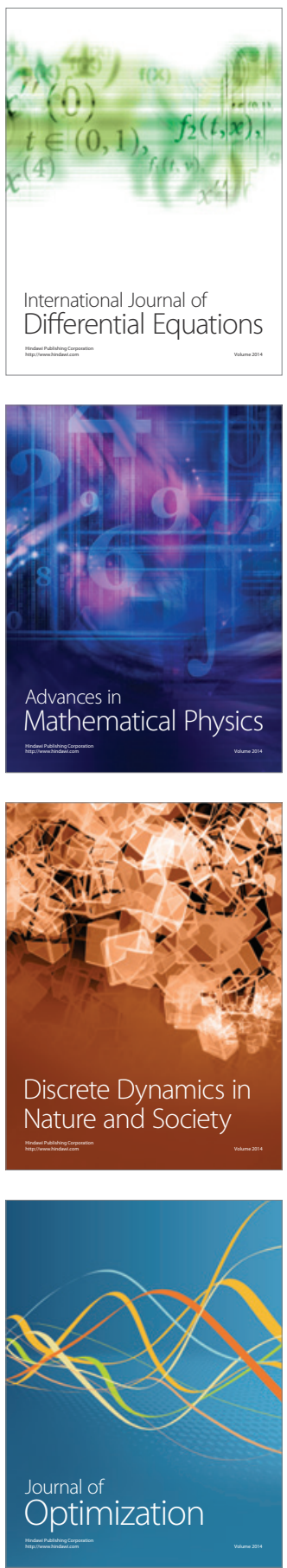